\title{
Comparative Analysis of Background Subtraction and CNN Algorithms for Mid-Block Traffic Data Collection and Classification
}

\author{
Ubaid Illahi \\ Department of Civil Engineering, \\ National Institute of Technology Srinagar, UT of Jammu \& Kashmir, India. \\ Corresponding author: ubaidillahi@ nitsri.net \\ Mohammad Shafi Mir \\ Department of Civil Engineering, \\ National Institute of Technology Srinagar, UT of Jammu \& Kashmir, India. \\ E-mail: shafi@nitsri.net
}

(Received May 27, 2020; Accepted August 12, 2020)

\begin{abstract}
Classification of vehicles in the traffic stream is a pre-requisite for planning and designing the facilities for road-users. Considering the importance and gaining popularity of automated systems in this field, the aim of this article is to compare two algorithms- one using the Background Subtraction (BS) technique and the other using Convolutional Neural Network (CNN) with a primary focus on an increased number of vehicle classifications. To check the reliability of these algorithms, the outputs produced were validated against the data obtained from Kachkoot Toll Plaza, India. The results were analyzed using drop-line diagrams and confusion matrices. The overall efficiency of the CNN-based algorithm (0.98) was found to be better than the BS-based algorithm (0.95). The comparison presented in this paper will be useful for transportation professionals and agencies.
\end{abstract}

Keywords- Computer vision, Traffic data, Object detection, Background subtraction, Convolutional neural network.

\section{Introduction and Background}

Vehicle classification forms the basis in almost all the transportation engineering and planning related works, for example, in the structural and geometric design of pavements, designing traffic regulatory and control devices, developing facilities for road-users, etc. Its accuracy affects the quality of the research, which in turn, affects the policies in the transportation sector (Illahi and Mir, 2020). The idea of grouping vehicles into classes is a way to distinguish them by their size, geometry, and planned application. Conventional approaches to vehicle counting and classification that include manual recordings such as observation sheets and semi-automatic devices such as traffic counting pads, etc. are still used. But these methods are tedious, highly error-prone, and require a huge amount of resources. Automated vehicle detection techniques are found to solve these issues and therefore, advantageous over conventional methods (Wang et al., 2017). It is noteworthy that some popular traffic flow models have also been validated using automated techniques (Kanagaraj et al., 2015). In recent years, owing to such benefits, automated techniques have drawn attention of the researchers as far as vehicle detection and classification is concerned. However, the use of automated devices like pneumatic sensor tubes, infrared/ laser scanners, etc. (Brosnan et al., 2015) are found to be less successful as these provide results that are either unclassified, expensive or both. Computer vision (CV) techniques in combination with machine learning (ML) tools, contrary to these, are gaining popularity in the detection and classification of vehicles in real-time traffic scenarios (Zaki et al., 2013). 
International Journal of Mathematical, Engineering and Management Sciences

Vol. 5, No. 6, 1440-1451, 2020

https://doi.org/10.33889/IJMEMS.2020.5.6.107

Apart from ML tools, there are broadly three CV techniques for the detection of moving objects; they are BS, temporal difference, and optical flow in which BS being the most popular (Daigavane et al., 2011). Zaki and Sayed (2018) found that vehicle detection and classification using CV techniques are highly sought-after techniques because of the ease with which data can be extracted and used for further analysis. The application of CV techniques and ML tools have been utilized efficiently under the umbrella of intelligent transportation systems. Saran and Sreelekha (2015) reviewed some important developments in ML tools such as neural network (NN) and its link with conventional classification systems such as probability estimation, tradeoffs involved, variable selection, etc. They found that NN is a competitive alternative to traditional classifiers. Similarly, Weinblatt et al. (2013) analyzed and reported length-based vehicle classification schemes and appropriate length bin boundaries. It is evident from the literature that the researchers have accepted the superiority of CV techniques and ML tools as vehicle classifiers. This has been demonstrated in a number of ways by identifying and classifying vehicles, pedestrians, the surrounding environment, etc. For example, in a study by Jocic et al. (2019), CNN was utilized to identify pedestrians, bicyclists, traffic lights, and cars.

The combination of CV techniques and ML tools work on the basis of image processing algorithms. These algorithms take a sequence of images from a camera or a recorded video in case of offline processing. Broadly, there are two vehicle detection and classification systems that are based on the way these carry out the detection and classification processes (Zaki and Sayed, 2018). On one hand, there exists a two-stage classification system in which the first stage detects the vehicles. In the second stage, the results obtained in the detection stage are further analyzed for vehicle classification (Yan et al., 2013). On the other hand, simultaneous detection and classification could also be achieved. In this system, the vehicle is detected and simultaneously tagged i.e. classified within a single stage.

The following are some of the vehicle detection and classification works successfully carried out using CV techniques, ML tools, or both: Zaki et al. (2013) developed an automated system for classifying road-users based on the discrimination of the shapes of the speed profiles of each roaduser type. Zhao and Nevatia (2003) used frame differencing and BS to detect passenger cars using the Bayesian network. Similarly, image features to segment motion were put to test by Daigavane et al. (2011). Buch et al. (2010) developed a model to detect and classify vehicles as well as roadusers. The model was tested in urban traffic conditions and validated from reference Imagery Library for Intelligent Detection System (i-LIDS) datasets from the UK Home Office. Müller et al. (2001) reviewed the classification algorithms of NN and proposed a conceptual framework for their use and selection. Khanloo et al. (2012) developed a framework that used a variety of appearance features with their parameters using ML. Li et al. (2017) proposed a unified framework for the simultaneous detection of bicyclists and pedestrians using the Upper Body Multiple Potentil Region (UB-MPR) and region CNN. Likewise, in a study by Wang et al. (2017), the vehicle classification system was developed using Faster R-CNN which was then tested on NVDIA Jetson TK1 board. Saran and Sreelekha (2015) proposed a vehicle detection and classification algorithm using Histograms of Oriented Gradients (HOG) and geometric features of the vehicles. Likewise, Yan et al. (2013) developed a CV-based algorithm to detect bicycles. Zaki and Sayed (2018) presented a road-user multi-step classification system in shared space facilities.

Some other methods/ techniques used by various researchers are also available in the literature. For example, George et al. (2013) developed an algorithm for automatic identification and classification of vehicles using acoustic signals. Wei et al. (2013) proposed an algorithm for 
International Journal of Mathematical, Engineering and Management Sciences

Vol. 5, No. 6, 1440-1451, 2020

https://doi.org/10.33889/IJMEMS.2020.5.6.107

identifying traffic phases using a K-means clustering and level of service method. Rajab et al. (2016) used a single-element piezoelectric sensor placed diagonally on a traffic lane in combination with ML technology to classify vehicles. Morris and Trivedi (2006) demonstrated simultaneous tracking and classification of vehicles in a real-time system monitoring highway traffic using cooperating tracker and classifier modules. Based on the observation of geometry of a bicycle i.e. a frame (in the form of two triangles) and two wheels (in the form of ellipses), a model was proposed by Lin and Young (2017) to detect bicycles in the traffic stream. Hu et al. (2014) proposed a real-time multiple bicycle detection algorithm which extracts a feature called multi-scale block local binary pattern (MBLBP). Harlow and Peng (2001) used "range sensors" to detect and classify vehicles.

Reviewing the literature, it is clear that the vehicle detection and classification are of importance in transportation designing and planning works. It was found that the algorithms based on CV techniques and ML tools have gained superiority over the traditional methods. The reasons are that these algorithms have proven to be more efficient and require less amount of resources. In these algorithms, the BS technique and $\mathrm{NN}$ were found to be most popular among the researchers. However, it was observed that only a fewer number of vehicle classifications have been attained using these algorithms. To meet the needs of transportation planners and transport agencies, more classifications are required to be attained in the practical field.

Considering the importance and gaining popularity of $\mathrm{CV}$ techniques in combination with ML tools for automatic traffic data collection and classification, the aim of this study is to compare two of the most popular algorithms with the primary focus on attaining a greater number of vehicle classifications. To achieve that, two algorithms- one based on the BS technique and the other based on CNN have been tailored to detect and classify eleven vehicle types within the same algorithm. These algorithms were then tested in the practical field which was followed by the validation of the results against the ground (true) data.

The remaining article is organized in the following manner: in Section 2, two traffic classification methodologies are presented and explained. This is followed by the explanation of the study area, data acquired, and classification of vehicles adopted in Section 3. Section 4 presents the results obtained from the site which is followed by elucidating and validating them. Finally, the article provides a succinct conclusion regarding the efficiency of the two algorithms in Section 5.

\section{Traffic Classification Methodologies}

In this study, two algorithms for vehicle detection and classification are compared. The algorithms are based on the BS technique (see Figure 1) and CNN (see Figure 4), respectively.

\subsection{Traffic Classification Algorithm using BS}

This approach consists of two fundamental stages: (1) vehicle detection using BS, (2) vehicle classification using a neural network. Initially, 500 frames of the video were used to train the background subtractor which then produces foreground masks of subsequent frames. These foreground masks contain all detected moving objects as blobs of pure white color due to thresholds. Then, contours are drawn around these detected blobs and apply certain validations to rule out obvious false positives such as the minimum size of the object in the frame. This produces a list of detected vehicles whose position is tracked with the help of centroid of the detected contour, also known as bounding-box (see Figure 2 and Figure 3) Once contours are drawn around various detected vehicles, a path of each vehicle is kept in memory. This path consists of the various 
International Journal of Mathematical, Engineering and Management Sciences

Vol. 5, No. 6, 1440-1451, 2020

https://doi.org/10.33889/IJMEMS.2020.5.6.107

positions of the centroid of the bounding box of the vehicle throughout the frames of the video that the vehicle is detected in.

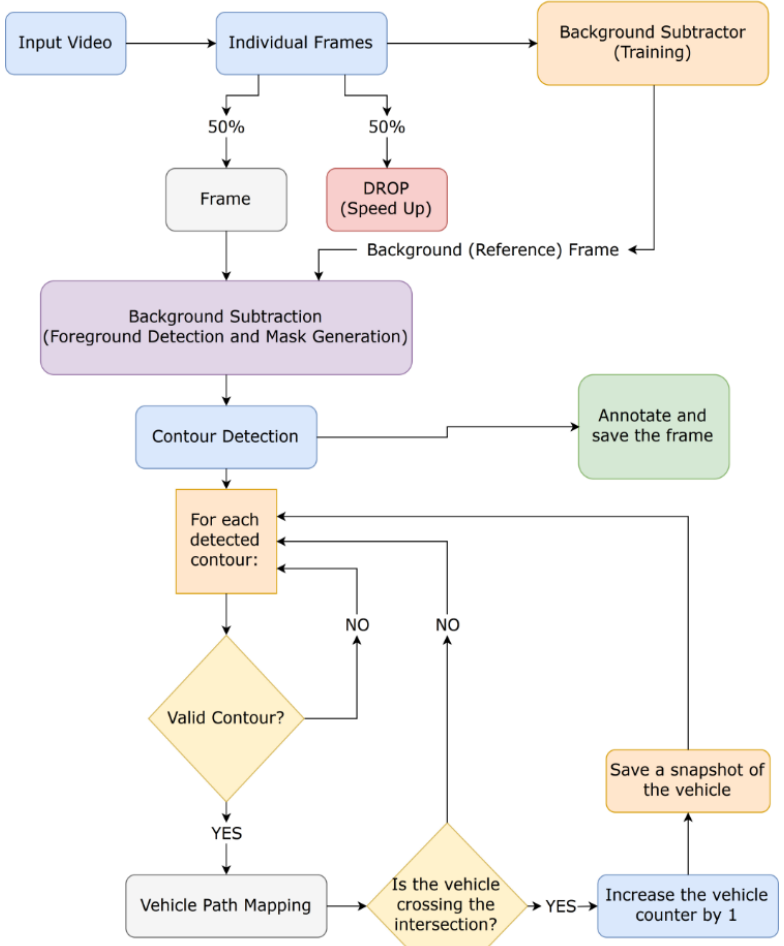

Figure 1. Mid-block traffic data collection algorithm using BS technique

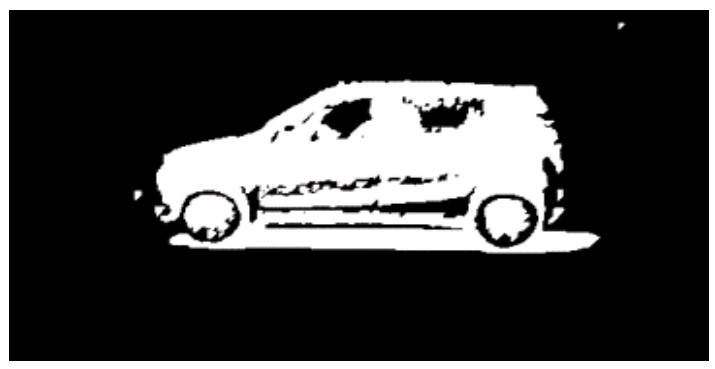

(a)

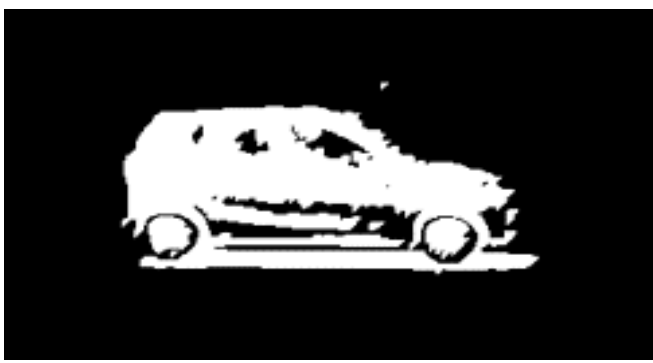

(b)

Figure 2. Extracted foreground masks of PPV classification run in BS algorithm (a) PPV moving from right to left, (b) PPV moving from left to right

\subsection{Traffic Classification Algorithm using CNN}

This approach consists of a pre-trained CNN-based system that detects and recognizes vehicles simultaneously within a frame. This system works on a pre-built classification system which then classifies the detected objects using inbuilt labels that the network has been trained upon. The process followed in this 
International Journal of Mathematical, Engineering and Management Sciences

Vol. 5, No. 6, 1440-1451, 2020

https://doi.org/10.33889/IJMEMS.2020.5.6.107

approach is presented in Figure 4. In this approach, the first stage consists of processing the frames through vehicle detection and recognition network with their respective coordinates and bounding boxes. These bounding boxes are used to locate and store the object in memory (see Figure 5). When the vehicle crosses the separation boundary, the counter for that specific class is increased by one in that particular direction.
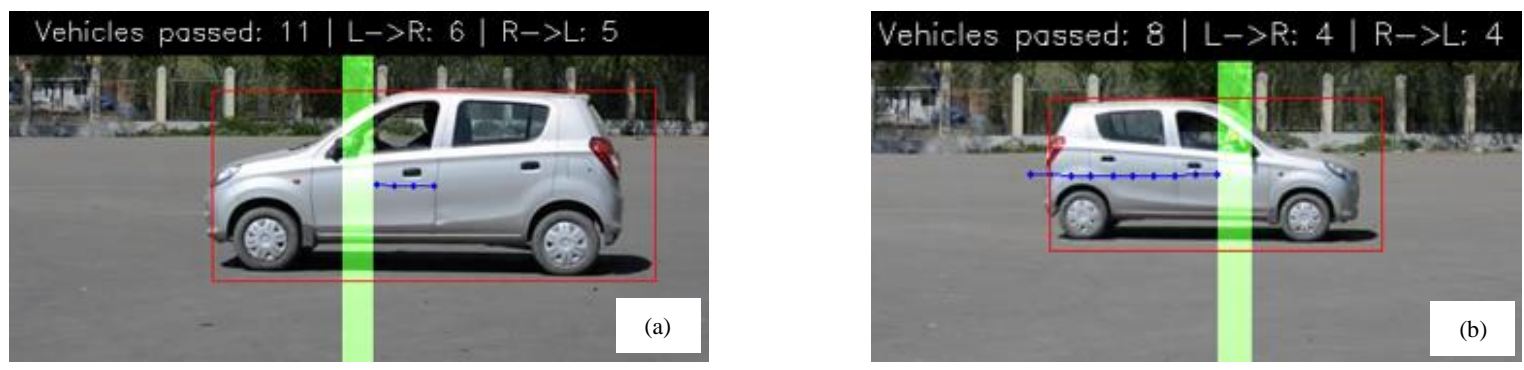

Figure 3. Bounding boxes of PPV classification run in BS algorithm (a) PPV moving from right to left, (b) PPV moving from left to right

\subsection{Required Adjustments}

Camera calibration is one of the vital steps in vehicle detection techniques involving video analysis applications. From side-view, overlapping vehicles that pass the boundary at the same instant of time are detected and counted as single "vehicle" or missed entirely. To overcome this problem, the detection and distinct-counting of the vehicles at the same instant in multi-lane/ multicarriageway is possible by adjusting the camera angle as well as its elevation. Keeping this in view, the camera was adjusted and calibrated as per the guidelines provided by Ismail et al. (2013).

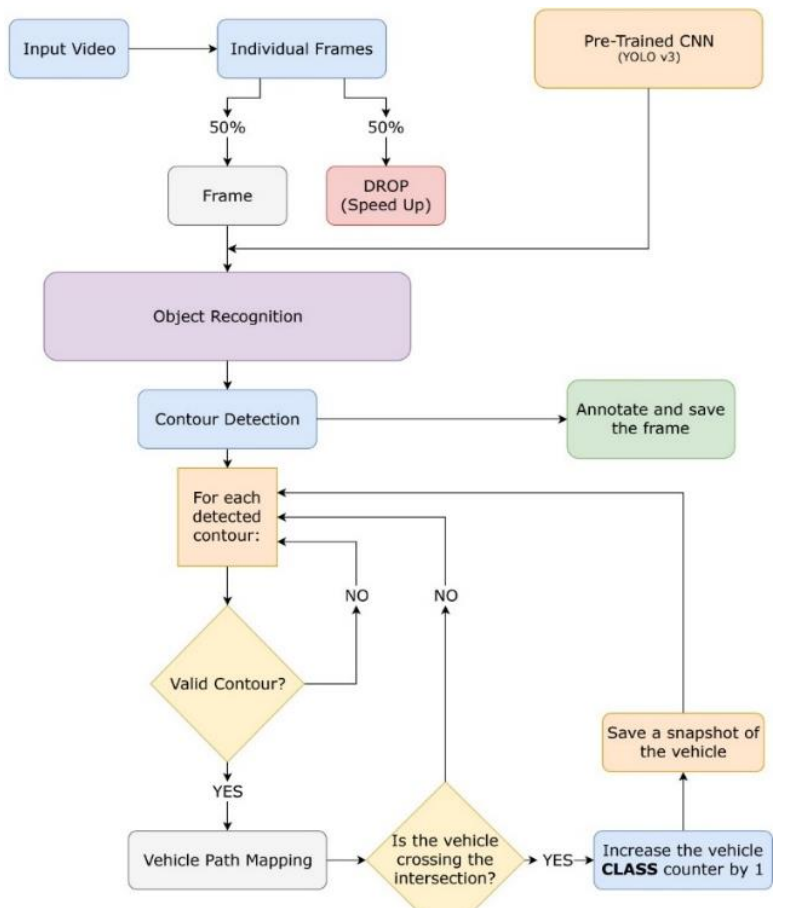

Figure 4. Mid-block traffic data collection algorithm using CNN 

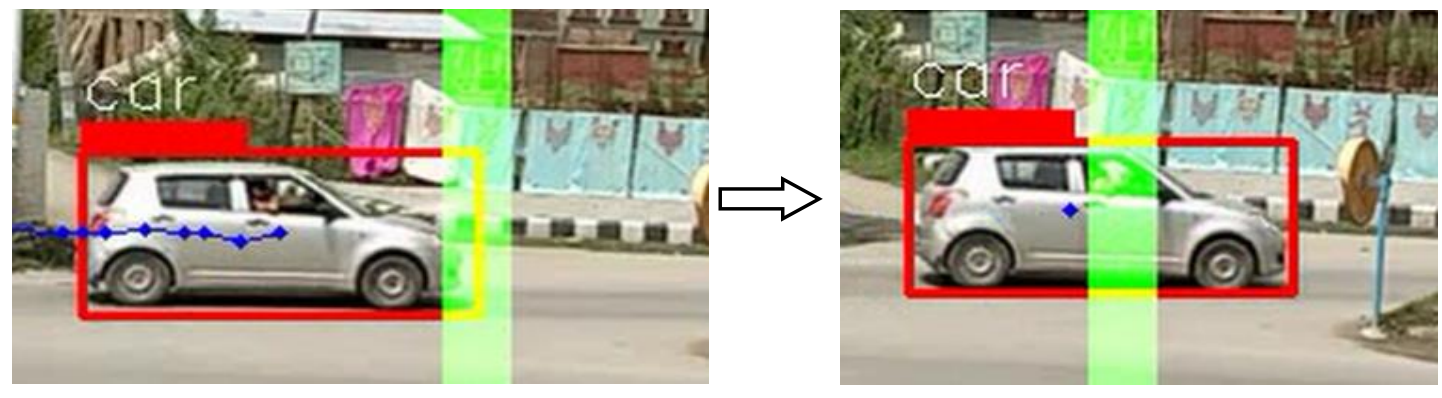

Figure 5. Simultaneous PPV detection and classification run in CNN algorithm

\section{Study Area and Vehicle Types}

Kachkoot Toll Plaza, which is located on NH-44, Amliar in the Union Territory (UT) of Jammu \& Kashmir was taken as the study area. 24-hour videos and data entry sheets from July 22, 2019 to July 28, 2019 were obtained from the concerned data processing department. A total of eleven vehicle classifications were considered in this study which includes 2 -wheelers, 3 -wheelers, private passenger vehicles (PPVs), light commercial passenger vehicles (LCPVs), light commercial goods vehicles (LCGVs), tractor-trailers, mini-buses, $\geq 2$-axle buses, 2-axle trucks, 3-axle trucks, and 46-axle trucks. It is important to mention that since no reliable data on the slow-moving nonmotorized vehicles such as bicycles, bullock-carts, pedestrians, etc. was available, these vehicle types were excluded from this study.

\section{Results, Validation and Discussion}

The one-week (24-hour) videos (as mentioned in Section 3) were run through both the BS-based and the CNN-based algorithms and the results obtained were validated against the data entry sheets provided by the concerned data processing department at Kachkoot Toll Plaza. The results for each of the eleven vehicle classifications are presented in Figure 6. To validate the results, various metrics were computed using the confusion matrices (refer to Table 1 and Table 2). The computed metrics include overall efficiency $(\eta)$, precision $(p)$, recall/ sensitivity $(\alpha)$ and specificity $(S)$ (refer to Equations 1-4).

$\eta=\frac{\sum T P}{\sum(T P+X)}$

$p=\frac{T P_{c}}{T P_{c}+F^{+}}$

$\alpha=\frac{T P_{c}}{T P_{c}+F^{-}}$

$S=\frac{T N_{c}}{T N_{c}+F^{+}}$

where, $T P$ and $T N$ are true positive and true negative, respectively; $F^{+}$and $F^{-}$are false positive and false negative, respectively; $X$ is the element in the confusion matrix other than the diagonal element; subscript $c$ stands for the corresponding class in the confusion matrix. 
International Journal of Mathematical, Engineering and Management Sciences

Vol. 5, No. 6, 1440-1451, 2020

https://doi.org/10.33889/IJMEMS.2020.5.6.107
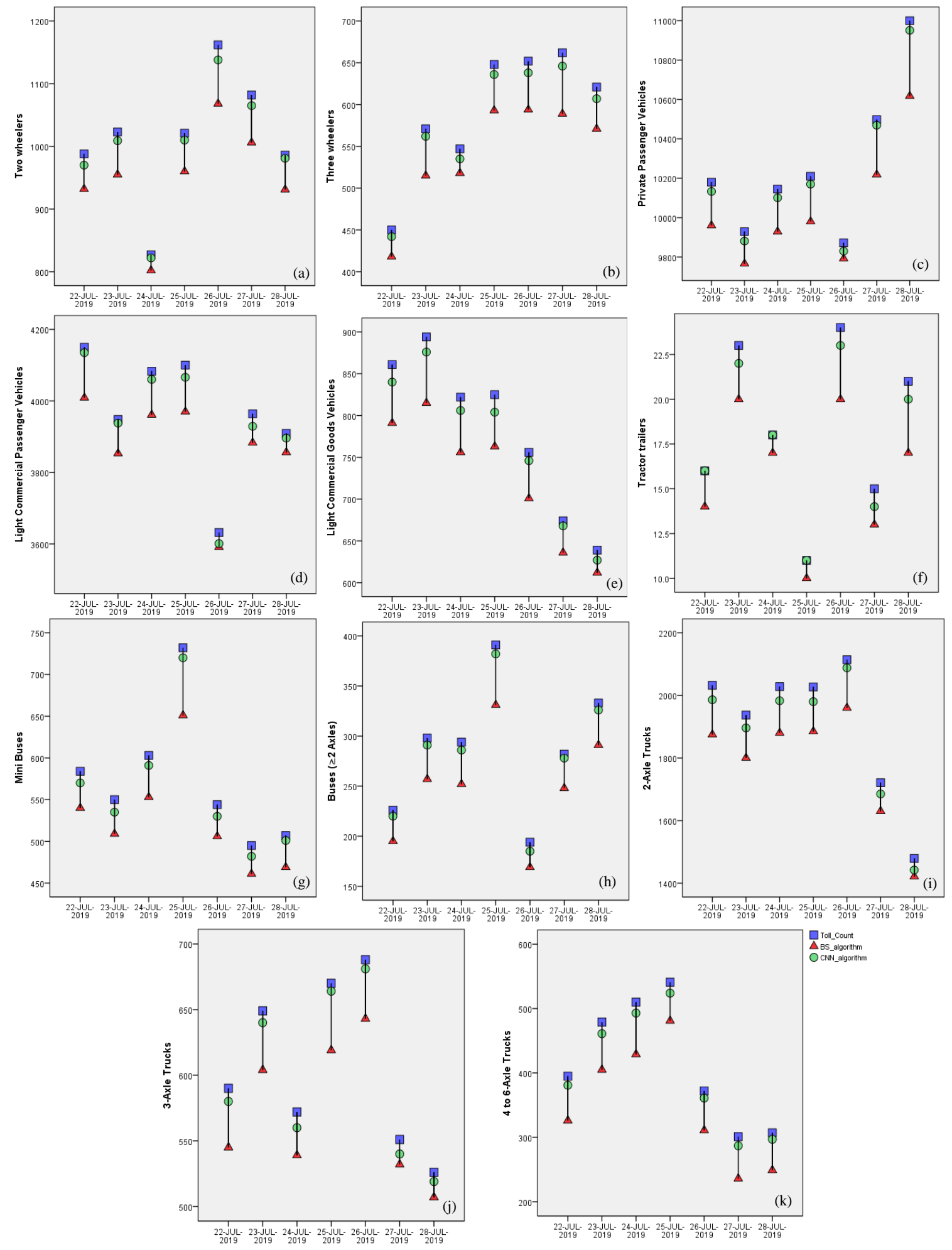

Figure 6. Drop-line diagrams for eleven vehicle classifications showing the comparison of BS-based and CNN-based algorithms with the Kachkoot Toll Plaza data 
International Journal of Mathematical, Engineering and Management Sciences

Vol. 5, No. 6, 1440-1451, 2020

https://doi.org/10.33889/IJMEMS.2020.5.6.107

In drop-line diagrams (Figure 6), it can be seen that, comparatively, the counts for all the eleven vehicle classifications obtained from the $\mathrm{CNN}$-based algorithm are close to the toll data. The confusion matrices (see Table 1 and Table 2) reflect the sub-metrics like true positive (TP), true negative $(\mathrm{TN})$, false positive $\left(\mathrm{F}^{+}\right)$and false negative $\left(\mathrm{F}^{-}\right)$, which in turn, are useful in obtaining the metrics like precision, specificity, sensitivity and overall efficiency of an algorithm. These metrics were computed for both the algorithms which were then plotted for all the eleven vehicle classifications (see Figures 7-9). These plots signify that the CNN-based algorithm is better in terms of the precision, specificity as well as the sensitivity in most of these vehicle classifications.

Also, considering the overall efficiency, the CNN-based algorithm performed better $(\eta=0.98)$ compared to the BS-based algorithm $(\eta=0.95)$ (see Figure 10).

Table 1. Confusion Matrix for BS-based algorithm

\begin{tabular}{|c|c|c|c|c|c|c|c|c|c|c|c|c|c|}
\hline \multirow[b]{2}{*}{ Toll count (Actual) } & $\begin{array}{l}\frac{n}{0} \\
\frac{\pi}{\mathbb{d}} \\
\frac{\pi}{3} \\
\frac{1}{d}\end{array}$ & $\begin{array}{l}\frac{n}{0} \\
\frac{0}{\mathbb{d}} \\
\frac{\pi}{3} \\
\frac{1}{n}\end{array}$ & $\vec{a}$ & 己ે & نِ & 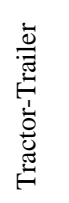 & 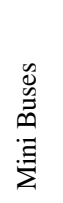 & $\begin{array}{l}0 \\
0 \\
0 \\
0 \\
0 \\
0 \\
\Delta \\
N \\
N\end{array}$ & 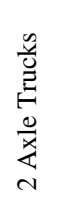 & $\begin{array}{l}\frac{\tilde{u}}{u} \\
\underline{\underline{E}} \\
\frac{u}{x} \\
m \\
m\end{array}$ & 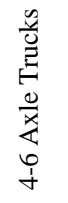 & \multirow[b]{2}{*}{$\mathrm{F}^{-}$} & \multirow[b]{2}{*}{ Count } \\
\hline & \multicolumn{11}{|c|}{ Output obtained from algorithm (predicted) } & & \\
\hline 2-wheelers & 0.94 & 0.06 & 0 & 0 & 0 & 0 & 0 & 0 & 0 & 0 & 0 & 0.06 & 7089 \\
\hline 3-wheelers & 0.09 & 0.91 & 0 & 0 & 0 & 0 & 0 & 0 & 0 & 0 & 0 & 0.09 & 4151 \\
\hline PPV & 0 & 0 & 0.97 & 0.02 & 0.01 & 0 & 0 & 0 & 0 & 0 & 0 & 0.02 & 71834 \\
\hline LCPV & 0 & 0 & 0.02 & 0.98 & 0.01 & 0 & 0 & 0 & 0 & 0 & 0 & 0.02 & 27786 \\
\hline LCGV & 0 & 0 & 0.02 & 0.05 & 0.93 & 0 & 0 & 0 & 0 & 0 & 0 & 0.07 & 5471 \\
\hline Tractor-Trailer & 0 & 0 & 0 & 0 & 0 & 0.87 & 0 & 0 & 0 & 0 & 0 & 0.13 & 128 \\
\hline Mini Buses & 0 & 0 & 0 & 0 & 0.08 & 0 & 0.92 & 0 & 0 & 0 & 0 & 0.08 & 4015 \\
\hline$\geq 2$ Axle Buses & 0 & 0 & 0 & 0 & 0 & 0 & 0 & 0.86 & 0 & 0.14 & 0 & 0.14 & 2018 \\
\hline 2 Axle Trucks & 0 & 0 & 0 & 0 & 0 & 0 & 0.05 & 0.02 & 0.93 & 0 & 0 & 0.07 & 13338 \\
\hline 3 Axle Trucks & 0 & 0 & 0 & 0 & 0 & 0 & 0 & 0.04 & 0 & 0.94 & 0.02 & 0.06 & 4246 \\
\hline 4-6 Axle Trucks & 0 & 0 & 0 & 0 & 0 & 0 & 0 & 0.11 & 0 & 0.05 & 0.84 & 0.16 & 2905 \\
\hline $\mathrm{F}^{+}$ & 0.09 & 0.06 & 0.04 & 0.07 & 0.09 & 0 & 0.05 & 0.17 & 0 & 0.32 & 0.02 & & \\
\hline
\end{tabular}

Note: The values in the confusion matrix are normalized between 0 and 1 .

Table 2. Confusion matrix for CNN-based algorithm

\begin{tabular}{|c|c|c|c|c|c|c|c|c|c|c|c|c|c|}
\hline \multirow[b]{2}{*}{ Toll count (Actual) } & 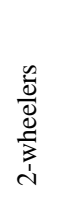 & 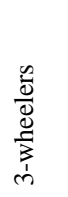 & $\vec{a}$ & 己े & ن્త & 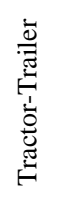 & 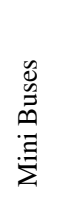 & 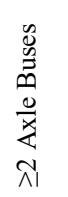 & 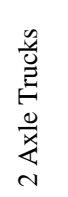 & $\begin{array}{l}\frac{n}{0} \\
\frac{0}{Z} \\
\frac{0}{x} \\
m\end{array}$ & 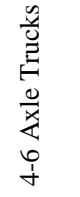 & \multirow[b]{2}{*}{$\mathrm{F}^{-}$} & \multirow[b]{2}{*}{ Count } \\
\hline & \multicolumn{11}{|c|}{ Output obtained from algorithm (predicted) } & & \\
\hline 2-wheelers & 0.99 & 0.01 & 0 & 0 & 0 & 0 & 0 & 0 & 0 & 0 & 0 & 0.01 & 7089 \\
\hline 3-wheelers & 0.02 & 0.98 & 0 & 0 & 0 & 0 & 0 & 0 & 0 & 0 & 0 & 0.02 & 4151 \\
\hline PPV & 0 & 0 & 0.99 & 0.01 & 0 & 0 & 0 & 0 & 0 & 0 & 0 & 0.01 & 71834 \\
\hline LCPV & 0 & 0 & 0 & 0.99 & 0 & 0 & 0 & 0 & 0 & 0 & 0 & 0.01 & 27786 \\
\hline LCGV & 0 & 0 & 0.01 & 0.01 & 0.98 & 0 & 0 & 0 & 0 & 0 & 0 & 0.02 & 5471 \\
\hline Tractor-Trailer & 0 & 0 & 0 & 0 & 0 & 0.97 & 0 & 0 & 0 & 0 & 0 & 0.03 & 128 \\
\hline Mini Buses & 0 & 0 & 0 & 0 & 0.02 & 0 & 0.98 & 0 & 0 & 0 & 0 & 0.02 & 4015 \\
\hline$\geq 2$ Axle Buses & 0 & 0 & 0 & 0 & 0 & 0 & 0 & 0.98 & 0 & 0.02 & 0 & 0.02 & 2018 \\
\hline 2 Axle Trucks & 0 & 0 & 0 & 0 & 0 & 0 & 0.01 & 0.01 & 0.98 & 0 & 0 & 0.02 & 13338 \\
\hline 3 Axle Trucks & 0 & 0 & 0 & 0 & 0 & 0 & 0 & 0.01 & 0 & 0.99 & 0 & 0.01 & 4246 \\
\hline 4-6 Axle Trucks & 0 & 0 & 0 & 0 & 0 & 0 & 0 & 0.02 & 0 & 0.01 & 0.97 & 0.03 & 2905 \\
\hline $\mathrm{F}^{+}$ & 0.02 & 0.01 & 0.01 & 0.02 & 0.02 & 0 & 0.01 & 0.04 & 0 & 0.07 & 0 & & \\
\hline
\end{tabular}

Note: The values in the confusion matrix are normalized between 0 and 1. 
International Journal of Mathematical, Engineering and Management Sciences

Vol. 5, No. 6, 1440-1451, 2020

https://doi.org/10.33889/IJMEMS.2020.5.6.107

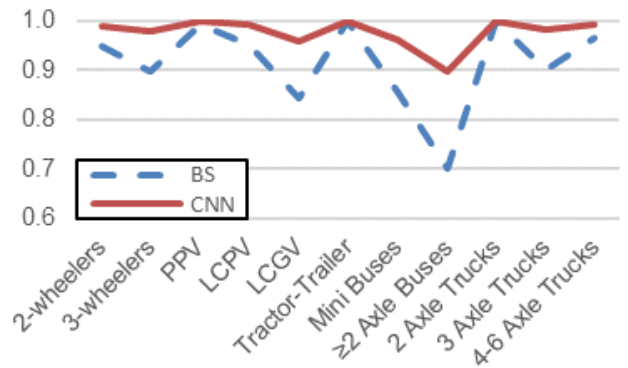

Figure 7. Precision curves of BS-based and CNN-based algorithms

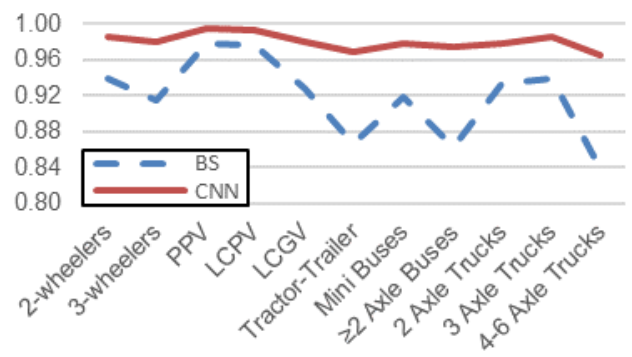

Figure 9. Recall/ Sensitivity curves of BSbased and $\mathrm{CNN}$-based algorithms

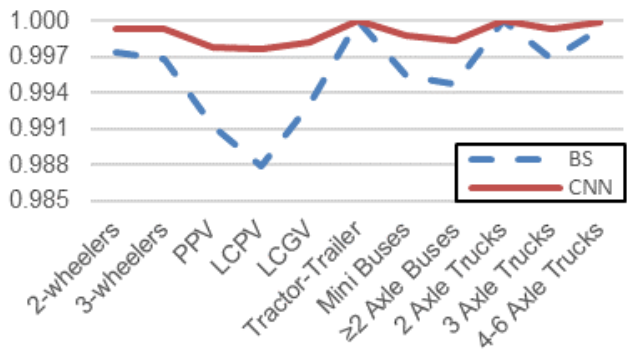

Figure 8. Specificity curves of BS-based and CNN-based algorithms

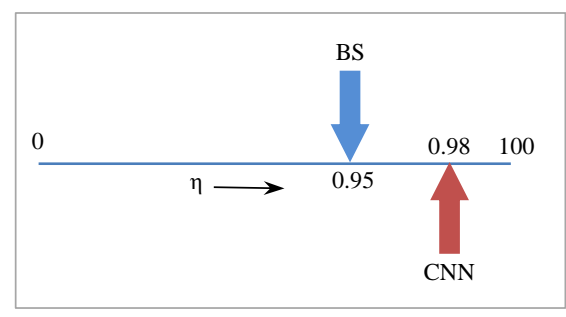

Figure 10. Overall efficiency of BS-based and CNN-based algorithms

Analyzing the results, some useful takeaways regarding the BS-based algorithm are as follows:

- In case the vehicles coming in the same or opposite direction cross the green vertical band simultaneously, the counting as well as the classification of the vehicles is adversely affected. This is mainly due to the vehicles overlapping in the frame.

- The overlapping issue can be dealt with by changing the view of the camera from side to elevated. The problem of vehicle counting, as a result of this, gets solved to an extent but, the accuracy of vehicle classification is reduced.

- Even slight physical disturbance to the camera due to environmental or human reasons causes vibrations and/ or shaking of the camera which creates noise in the foreground mask and therefore reduces the efficiency of vehicle detection.

- Comparatively, this algorithm of vehicle detection and classification is time-consuming. However, it requires lesser computational power.

Some valuable takeaways regarding the $\mathrm{CNN}$-based algorithm are as follows:

- The vehicle detection, in this case, is free from any foreground mask and is unaffected by the vibrations or shaking of the camera. Therefore, the accuracy of detecting and classifying vehicles is increased. 
International Journal of Mathematical, Engineering and Management Sciences

Vol. 5, No. 6, 1440-1451, 2020

https://doi.org/10.33889/IJMEMS.2020.5.6.107

- A suitable camera angle can be used for collecting data, although making sure that the vehicles must be visible and distinguishable. Moreover, no background training is required in this case.

- This algorithm proves to be more efficient in terms of computational power required on aggregate and time taken for simultaneous object detection and classification of vehicles.

- The object detection and classification of vehicles are done simultaneously and therefore the whole process becomes simple and less tedious.

\section{Conclusions}

The aim of this study was to compare two algorithms for automatic detection and classification of vehicles on mid-block road sections with a primary focus on an increased number of classifications. Therefore, BS-based and CNN-based algorithms were tailored and then tested. The results from both the algorithms were validated against Kachkoot Toll data using drop-line diagrams and confusion matrices. Analyzing the results showed that the CNN-based algorithm outperformed the BS-based algorithm in terms of precision, specificity and recall/ sensitivity. The overall efficiency of the CNN-based algorithm $(\eta=0.98)$ was also found to be better as compared to the BS-based algorithm $(\eta=0.95)$. Moreover, considering the overall time, the $\mathrm{CNN}$-based algorithm requires less computational power. Considering these merits, this algorithm will be helpful to transportation agencies and professionals.

\section{Conflicts of Interest}

The authors confirm that there is no conflict of interest to declare for this publication.

\section{Acknowledgment}

The authors would like to thank the Project Director PIU Srinagar, National Highway Authority of India, Ministry of Road Transport and Highways, Government of India for providing the video-recordings and punched data of Kachkoot Toll Plaza (NH-44).

\section{References}

Brosnan, M., Petesch, M., Pieper, J., Schumacher, S., \& Lindsey, G. (2015). Validation of bicycle counts from pneumatic tube counters in mixed traffic flows. Transportation Research Record, 2527(1), 80-89. https://doi.org/10.3141/2527-09.

Buch, N., Orwell, J., \& Velastin, S.A. (2010). Urban road user detection and classification using 3D wire frame models. IET Computer Vision, 4(2), 105-116. https://doi.org/10.1049/iet-cvi.2008.0089.

Daigavane, P.M., Bajaj, P.R., \& Daigavane, M.B. (2011). Vehicle detection and neural network application for vehicle classification. In International Conference on Computational Intelligence and Communication Systems (pp. 758-762). IEEE. Gwalior, India. https://doi.org/10.1109/CICN.2011.168.

George, J., Mary, L., \& Riyas, K.S. (2013). Vehicle detection and classification from acoustic signal using ANN and KNN. In International Conference on Control Communication and Computing (pp. 436-439). IEEE. Thiruvananthapuram, India. https://doi.org/10.1109/ICCC.2013.6731694.

Harlow, C., \& Peng, S. (2001). Automatic vehicle classification system with range sensors. Transportation Research Part C: Emerging Technologies, 9(4), 231-247. https://doi.org/10.1016/S0968090X(00)00034-6. 
International Journal of Mathematical, Engineering and Management Sciences

Vol. 5, No. 6, 1440-1451, 2020

https://doi.org/10.33889/IJMEMS.2020.5.6.107

Hu, H., Tao, P., Gao, Z., Wang, Q., Li, Z., \& Qu, Z. (2014). Vision-based bicycle detection using multiscale block local binary pattern. Mathematical Problems in Engineering, 2014(Special), 1-7. https://doi.org/10.1155/2014/370685.

Illahi, U., \& Mir, M.S. (2020). Sustainable transportation attainment index: multivariate analysis of indicators with an application to selected states and National Capital Territory (NCT) of India. Environment, Development and Sustainability, Article in press. https://doi.org/10.1007/s10668-020-00734-w.

Ismail, K., Sayed, T., \& Saunier, N. (2013). A methodology for precise camera calibration for data collection applications in urban traffic scenes. Canadian Journal of Civil Engineering, 40(1), 57-67.

Jocic, D., Cirovic, V., \& Aleksendric, D. (2019). Identification and recognition of vehicle environment using artificial neural networks. Lecture Notes in Networks and Systems, 54, 208-219. https://doi.org/10.1007/978-3-319-99620-2_16

Kanagaraj, V., Asaithambi, G., Toledo, T., \& Lee, T.C. (2015). Trajectory data and flow characteristics of mixed traffic. Transportation Research Record, 2491(1), 1-11. https://doi.org/10.3141/2491-01.

Khanloo, B.Y.S., Stefanus, F., Ranjbar, M., Li, Z.N., Saunier, N., Sayed, T., \& Mori, G. (2012). A large margin framework for single camera offline tracking with hybrid cues. Computer Vision and Image Understanding, 116(6), 676-689. https://doi.org/10.1016/j.cviu.2012.01.004.

Li, X., Li, L., Flohr, F., Wang, J., Xiong, H., Bernhard, M., Pan, S., Gavrila, D.M., Li, K. (2017). A unified framework for concurrent pedestrian and cyclist detection. IEEE Transactions on Intelligent Transportation Systems, 18(2), 269-281.

Lin, Y.B., \& Young, C.P. (2017). High-precision bicycle detection on single side-view image based on the geometric relationship. Pattern Recognition, 63(March), 334-354. https://doi.org/10.1016/j.patcog.2016.10.012.

Morris, B., \& Trivedi, M. (2006). Improved vehicle classification in long traffic video by cooperating tracker and classifier modules. In IEEE International Conference on Advanced Video and Signal Based Surveillance. IEEE. Sydney, Australia. https://doi.org/10.1109/AVSS.2006.65.

Müller, K.R., Mika, S., Rätsch, G., Tsuda, K., \& Schölkopf, B. (2001). An introduction to kernel-based learning algorithms. IEEE Transactions on Neural Networks, 12(2), 181-201.

Rajab, S., Kalaa, M.O.Al., \& Refai, H. (2016). Classification and speed estimation of vehicles via tire detection using single-element piezoelectric sensor Samer. Journal of Advanced Transportation, 50(7), $1366-1385$.

Saran, K.B., \& Sreelekha, G. (2015). Traffic video surveillance: vehicle detection and classification. In International Conference on Control, Communication and Computing India (pp. 516-521). IEEE. Trivandrum, India. https://doi.org/10.1109/ICCC.2015.7432948.

Wang, X., Zhang, W., Wu, X., Xiao, L., Qian, Y., \& Fang, Z. (2017). Real-time vehicle type classification with deep convolutional neural networks. Journal of Real-Time Image Processing, 16(1), 5-14. https://doi.org/10.1007/s11554-017-0712-5.

Wei, H., Liu, H., Ai, Q., Li, Z., Xiong, H., \& Coifman, B. (2013). Empirical innovation of computational dual-loop models for identifying vehicle classifications against varied traffic conditions. ComputerAided Civil and Infrastructure Engineering, 28(8), 621-634. https://doi.org/10.1111/mice.12015.

Weinblatt, H., Minge, E., \& Petersen, S. (2013). Length-based vehicle classification schemes and length bin boundaries. Transportation Research Record, 2339(1), 19-29. https://doi.org/10.3141/2339-03.

Yan, J., Ling, Q., Zhang, Y., Li, F., \& Zhao, F. (2013). An adaptive bicycle detection algorithm based on Multi-Gaussian models. Journal of Computational Information Systems, 9(24), 10075-10083. https://doi.org/10.12733/jcis8668. 
International Journal of Mathematical, Engineering and Management Sciences

Vol. 5, No. 6, 1440-1451, 2020

https://doi.org/10.33889/IJMEMS.2020.5.6.107

Zaki, M.H., \& Sayed, T. (2018). Automated class identification of modes of travel in shared spaces: a case study from India. IET Intelligent Transport Systems, 12(8), 765-773. https://doi.org/10.1049/ietits.2017.0099.

Zaki, M.H., Sayed, T., \& Mori, G. (2013). Classifying road users in urban scenes sing movement patterns. Journal of Computing in Civil Engineering, 27(4), 395-406. https://doi.org/10.1061/(ASCE)CP.

Zhao, T., \& Nevatia, R. (2003). Car detection in low resolution aerial images. Image and Vision Computing, 21(8), 693-703. https://doi.org/10.1016/S0262-8856(03)00064-7. 